\title{
KCNQ10T1 Knock Down Alleviates Hyperosmlity Induced Dry Eye Disease via Inhibiting NLRP3 Inflammsome Activation and IL-1 $\beta$ Release
}

\section{Xuran Li}

First Affiliated Hospital of Harbin Medical University

\section{Yanyan Zhang}

First Affiliated Hospital of Harbin Medical University

\section{Xinyue Li}

First Affiliated Hospital of Harbin Medical University

Yu Yu

First Affiliated Hospital of Harbin Medical University

\section{Xin Jin}

First Affiliated Hospital of Harbin Medical University

Hong Zhang ( $\nabla$ zhanghong@hrbmu.edu.cn )

First Affiliated Hospital of Harbin Medical University

\section{Research Article}

Keywords: hyperosmlity, dry eye disease, inflammsome, activation, inflammatory diaeases, age, pathological changes

Posted Date: August 2nd, 2021

DOI: https://doi.org/10.21203/rs.3.rs-721954/v1

License: (c) (i) This work is licensed under a Creative Commons Attribution 4.0 International License. Read Full License 


\section{Abstract}

Background. Long noncoding RNAs (IncRNAs) have been indicated that participate in inflammatory diaeases and age related diseases by regulating physiological and pathological processes directly or indirectly. Evidences have indicated IncRNAs might be involving in dry eye disease which can cause pathological changes of cornea and conjunctiva in ocular surface though inflammatory response. KCNQ10T1 is a novel IncRNA, whose function remains totally unclear.

Methods. qRT-PCR was performed to detect the expression of KCNQ10T1, miR-214 and inflammasomerelated genes NLRP3, caspase 1 and IL-1 $\beta$. Western blotting was carried out to detect inflammasomerelated markers. The dry eye disease (DED) model was set up by scopolamine hydrobromide though subcutaneous injection. Bioinformatics was used to predict and validate the interaction between KCNQ10T1 and miR-214 as well caspase1 and miR-214.

Results. KCNQ10T1 was significantly up-regulated during the process of DED while miR-214 was contrarily down-regulated. Knockdown of KCNQ10T1 promoted cornea epithelial cells migration and down-regulated inflammasome-related genes. It was also confirmed that KCNQ10T1 directly interacted with miR-214. Meanwhile, miR-214 could bind to 3'UTR of caspase1 and therefore inhibited its expression. Furthermore, co-transfection of miR-214 inhibitor could rescue the down-regulation of cell migration induced by KCNQ10T1 knockdown.

\section{Introduction}

Dry eye disease (DED) been defined as one of the most common ocular dysfunctions caused by a combination of tear and ocular surface factors, including a range of symptoms such as pain, visual disturbances, tear film instability, even blindness[1]. Among all the pathological progresses of DED, the increase of osmolarity damage corneal and conjunctival epithelial cell and induce inflammation responses. Recently years $\triangle D E D$ has been recognized that an inflammatory disease because of the presence of inflammation-related factors, including IL-1b,IL-6,IL8,IFN-c,MMP9,TNF-a appeared in tear fluids collected from patients[2].The corneal epithelium cells (HCECs) contained in tears serves as the crucial defence against endogenous and exogenous pathogeny, also released pro-inflammatory interleukin-18 and interleukin-1b when exposed in the hyperomosity environment during in vitro assays.As we all know, the maturate of proinflammatory cytokines IL-1ßand IL-18 could be mediated by the activation of caspase-1, which recruited by inflammasome[3].

Inflammasomes is an intracellular multiprotein complex, and the assemble requires cytosolic sensing of pathogen-associated molecular patterns or danger-associated molecular patterns by a nucleotide-binding domain and leucine-rich repeat receptor (NLR) or absent in melanoma 2 (AIM2)-like receptors (ALR), further plays a critical role as a regulator of the innate immunity of the host defense against inflammatory diseases with various other type, such as NLRP3, NLRP1, AIM2, NLRC4[4]. 
Among them, canonical NLRP3 inflammasome has been recognized associated with several ocular diseases, such as human autoimmune disease, infectious disease [5]. Additionally, NLRP3 inflammasome has been comfrimed to participate in HCECs and conjunctival epithelial cells in paitents with DED, another indication is that ROS-induced oxidative stress might cause NLRP3 inflammasome activation in a conditional desiccating stress -induced murine model $[6,7]$.

Recently, a number of mechanisms have been proposed, including mitochondrial-associated dysfunction, oxidized mitochondrial DNA, cardiolipin translocation, potassium efflux, however, non-coding RNAs (ncRNAs) were not found to be a factor to activate NLRP3 inflammasome activation during DED. Human genome are transcribed into RNAs, and non-coding RNA account for approximately $90 \%$. For a long time, gene regulatory function has been mainly focus on protein coding genes, whereas Long-stranded noncoding RNAs (IncRNAs)is considered as a single-stranded RNA and also play critical regulatory roles in multiple biological processes though gene epigenetics, transcription and post-transcription regulation although it does not encode and be translated into proteins.

KCNQ10T1 is one of the important regulatory IncRNAs involved in cell cycle differentiation, development, senescence and other biological processes as well as human cancer[8],Autoimmune diseases, inflammatory diseases [9], neovascularization [10] and has been also reported involved in ophthalmic diseases.The expression level of KCNQ10T1 was upregulated and correlated with diabetic retinopathy stage and visual function loss [11], and can be triggered in diabetic human and rat corneal endothelium, along with the high glucose-treated corneal endothelial cells, also among macular oedema[12],corneal neovascularization[13].The most common mechanism for IncRNAs being mRNA regulatory factor is act as a competing endogenous RNA of miRNAs.KCNQ10T1 also can be bound microRNA to act as ceRNA to form a protective effect on target mRNA.

MicroRNAs (miRNAs) are another set of noncoding RNAs which has a short nucleotide sequence that can damage mRNA stability, affect transcription, and intervene translation by partially or completely combining with the 3'-UTR of their targeted mRNAs. For example, MiR-214 could simultaneously bind to 3'UTR of IncPVT1, TP53 or TFR1 regulated ferroptosis in brain ischemia/reperfusion (I/R) in vivo and in vitro [14]. MiR-214 was determined to participate to control pathological perivascular fibrosis in hypertension mediated by $T$ cell recruitment and local profibrotic cytokine release[15]. It was reported that miR-214 inhibit bone formation via targets ATF4 in osteoporosis. The binding site for KCNQ10T1 and miR-214 was predicted by bioinformatics analysis. And we previous has recommend that the increase in miR-214 after KCNQ10T1 knockdown could downregulate the NLRP3 inflammasome activation in diabetic cornea endothelial cells.

Thus, we hypothesized that KCNQ10T1 might function by targeting miR-214 in the aggression of pathological process during DED. We detected KCNQ10T1, miR-214, expression in tissues and cells after DED model establishment. In addition, we explored the involvement of the KCNQ10T1,miR-214,and NLRP3 inflammasome and explain concretely mechanisms that influence cell viability and inflammation in DED. 
These results may elucidated that KCNQ10T1 acted as molecular regulation in DED, provide a novel understanding of pathomechanism and offer an alternative approach to treat DED

\section{Materials \& Methods}

Animal model

DED was induced by placing the rats in a controlled environment with a relative humidity below $13 \%$ for consecutive fourteen days. To achieve maximum ocular surface dryness, rats in the controlled environment were injected subcutaneously $0.1 \mathrm{ml}$ scopolamine hydrobromide every four times per day. At day 3, 7, and 14 after DED induction, the corneal staining scoring was perform. Protocols were approved by the Harbin Medical University Animal Care and Use Committee guideline, which accords with Helsinki Declaration (2013 version). Weight from 160-180g male SD rats were purchased from the animal experiment center of the Second Affiliated Hospital of Harbin Medical University. The sex has no influence on experiment.

Corneal fluorescein staining

Briefly, corneal fluorescein staining was examined with a slit lamp microscope after dropping $1 \%$ $2 \%$ sodium fluorescein into the conjunctival sac of the lower fornix. After $1 \sim 2$ minutes, the corneal epithelium defect was stained with yellow-green color. The evaluation was taken according Oxford method[16].

Cell culture and Cell Transfection

HCECs were cultured in Dulbecco's modified DMEM/F12 supplemented with $10 \%$ foetal bovine serum (FBS) at $37^{\circ} \mathrm{C}$ in a $5 \% \mathrm{CO} 2$ overnight, or were treated with the different concentrations of sodium chloride solution for the additional appropriate time.

SiKCNQ10T1, miR-214 mimics, miR-214 inhibitors and their negative controls were obtained from RIBOBIO (Guangzhou, China). All transfections were carried out according to the manufacturer's protocol.

Cell scratch assay

After the cells are uniformly covered at the bottom of the 12-well plate, the marker pen is used to draw a horizontal line uniformly at the back. Part of the cells were removed with $200 \mu$ pipette perpendicularly to the horizontal line. After washing with PBS for three times, photos were taken at regular intervals and analysed by Image Studio software.

PCR

Total RNA was adopted refer to standard methods using TRIzol reagent (Invitrogen, Carlsbad, CA, USA). PCR primers were purchased from Invitrogen (Carlsbad, CA). The concentration of RNA was determined using a NanoDrop Spectrophotometer (NanoDrop Technologies, Wilmington, DE). 
Quantitative real-time PCR was performed using SYBR Green Real-Time PCR MasterMix (ToYoBo, Japan) according to the manufacturer's protocols using an ABI 7500 Sequence Detection System (Life Technologies, NY, USA). PCR conditions were as follows: holding $95^{\circ} \mathrm{C}$ for $60 \mathrm{~s}, 40$ cycles at $95^{\circ} \mathrm{C}$ for $15 \mathrm{~s}$, $60^{\circ} \mathrm{C}$ for $15 \mathrm{~s}, 72^{\circ} \mathrm{C}$ for $45 \mathrm{~s}$. The relative RNA expression levels were calculated by the $2-\triangle \triangle \mathrm{Ct}$ method.

Western-blot

After the total protein was extracted, the standard curve of the protein was prepared. BCA method was used for coloration analysis and calculation of the protein concentration on a biological spectrophotometer (Beyotime, China). SDA-PAGE gel was used for electrophoresis, then the protein was transferred to PVDF membrane, followed by milk blocking, primary antibody incubation, fluorescent secondary antibody incubation, and then immunoreactivity was detected with the Odyssey fluorescent scanning system (LI-COR) and analysed by Image Studio software.

Statistical analysis

Graph plots from experimental results and data analysis were carried out using GraphPad Prism Software 6.0 (GraphPad Software, La Jolla, USA). Results from experimental data were expressed as mean \pm standard deviation. $P$ values less than 0.05 were considered to be significant.

\section{Results}

1. NLRP3 inflammasome was induced in dry eye disease both in vivo and in vitro

Tear hyperosmotity is an important index during the dry eye disease. To investigate the effects of DED on cornea, we use a scopolamine hydrobromide subcutaneously injection model to destroy the lachrymal gland cell function, then destroy the stability of tear film to simulate the ocular microenvironment during DED.

To determine whether corneal tissue has been damage in induced DED, the corneal irregularity and staining score were measured by using sodium fluorescein囚the corneal epithelial presents spot and flake fluorescent staining (1A-B), indicating of the integrity of corneal epithelium was destructed.

In previous studies, NLRP3 inflammasome and inflammatory reactions have been reported to present to environment induced DED cornea tissues, therefore, qRT-PCR was performed to determine if they also exist in the cornea tissue of scopolamine hydrobromide injection rats. The DED group showed a remarkable increase in the mRNA expression of IL-1 $\beta$, NLRP3 and caspase-1 which consist of inflammsome compared with the control rats receive PBS injection (1C-E).

Meanwhile, protein data showed that NLRP3 expression significantly increased in the DED group, and its downstream factors, caspase- 1 , IL-1 $\beta$, are elevated (1F-G). 
We further confirmed the effect of DED on NLRP3 inflammasome in vitro by hyperosmolarity-induced HCECs, and the cell vitality has a significantly decrease when treated with sodium chloride $(\mathrm{NaCl}, 500$ mOsM) for 24h.qRT-PCR showed that cultured with the hyperosmotic media significantly increased the mRNA expression of IL-1 $\beta$, NLRP3 and caspase-1 $(1 \mathrm{H})$, in addition, the protein expression of IL-1 $\beta$, NLRP3 and caspase- 1 also have a significantly increased by hyperosmotic stress $(\mathrm{I}, \mathrm{J})$.

2. KCNQ10T1 was upregulated in DED and recede the growth of HCECs

Recent studies have indicated that KCNQ10T1 expression upregulated in metabolic disease [17]. To evaluate the content of KCNQ10T1 in DED, KCNQ10T1 expression validated by PCR was also higher in HCECs in hyperosmoity conditions compared with that controls (2A). Moreover, the expression analysis verified that there was significantly increased in cornea tissue collected from DED mouse (2B).

To investigate the effects of KCNQ10T1 on migration of HCECs, we conducted siRNA sequence (siKC) or scrambled siRNA (siNC) to reduce the expression of KCNQ10T1. After 48 hours transfection, the migration rate of HCECs was increased after KCNQ10T1 was knocked down compared with the negative control (2C-D).

The role of KCNQ10T1 how participate in mediating NLRP3 inflammasome which induced in DED still unclear. To determine whether siKC has an alleviating effect on DED-induced HCECs damage,

Therefore, PCR was performed to determine the anti-inflammatory effect, the hyperosomity induced group showed a remarkable decrease in the mRNA expression of NLRP3 and caspase-1, mRNA expression of IL$1 \beta$ also decreased (2E-G).

Treatment with siKC also have a decrease effect on the protein expression level of NLRP3, caspase-1 and $\mathrm{IL}-1 \beta(2 \mathrm{H}-\mathrm{I})$.

Make a conclusion, these data suggested that knock down the expression of KCNQ10T1 decreased the expression of inflammatory cytokines by inhibiting the NLRP3 inflammasome pathway in vitro.

3. MiR-214 mimics rescued the cellular response by targeting caspase-1

In PCR analysis, we found the level of miR-214 was also significantly downregulated in HCECs treated with hyperosmoity media(3A), which has an coherence with DED mouse cornea tissue compared with healthy controls(3B).

Regarding TargetScan and miRbase prediction, miR-214 were identified as the most significant one to potential for binding on the $3^{\prime}$ UTR of caspase-1(3C).

We focused on the counteract occurs in between miR-214 and caspase- 1 ,following the change with miR214 level, overexpression of miR-214 significantly suppressed mRNA, while HCECS showed significantly higher expression after miR-214 inhibitors transfection(3E),while the protein expression of caspase-1 within HCECs have an accordance with mRNA content after exposed to hyperosmoity media (3D,3F). 
The correlation between the expression pattern of miR-124 and cell migration was determined, high expression of miR-214 lead to a higher migrate rate in HCECs compared with those incubated with hyperosmoity media(3G-H).

4. KCNQ10T1 should mediated the apoptosis, growth and migration of HCECs being a sponge of miR214

It has been clarified that IncRNA may act as a kind of ceRNA of miRNA. To further explore the potential mechanism of KCNQ10T1 in DED, bioinformatics analysis was used predict and identify the target genes of miR-214(4A).The relationship exist between KCNQ10T1 and miR-214 was determined by transfecting siKC, qPCR manifested

the expression of miR-214 was higher than that $\operatorname{siNC}(4 \mathrm{~B})$.

To explore the effects of the interaction between KCNQ10T1 and miR-214 in HCECs, siKC+miR-214 mimic and siKC+ miR-NC were transfected

The decline of miR-214 partly reversed the influence of KCNQ10T1 on cell migration (4C-D), this confirmed that KCNQ10T1 regulate HCECs during DED by sponging miR-214.

In summary, the results showed that the expression of KCNQ10T1 and miR214 were negatively correlated, and the expression of miR214 and caspase- 1 were negatively correlated, while the expression of KCNQ10T1 and caspase-1 were positively correlated during the disease process.

\section{Discussion}

Herein, we first provide novel evidence for the interaction between KCNQ10T1, miR-214 and caspase1 during DED,NLRP3 inflammsome activation and IL-1 $\beta$ release were involved in corneal epithelial stress response, and KCNQ10T1 knock down promotes HCECs migration and alleviates cell death by sponging miR-214 though downregulating expression of caspase-1.

The microenvironment change of ocular surface which consisted of the cornea, conjunctiva, lacrimal glands, and connective tissue could come from an imbalance of tear film stability and osmolarity. Tear membrane stability contribute to lubrication and moisture cornea to surive from dryness and irritation [18], which characterized as DED.

The pathogenesis contributed to DED have been shown multifactorial, oxidation, inflammation, and cell apoptosis all participation constitutes to deteriorate the process.

Until now, several animal models have been used to investigate DED, and we used lacrimal glanddamaged rats to explore the pathology of DED. In this study, Fluorescein sodium staining confirmed the damage of the cornea epithelium exist in DED animal models induced by tear deficiency 
The inflammatory cytokines release is related with the ocular surface epithelial cells dysfunction. For example, IL-1 and IFN- $\gamma$ make squamous metaplasia and IFN- $\gamma$ influencce goblet cell differentiation [19].

In our study, we estimate the in vivo and in vitro inflammatory factors expressed upon the DED, and found there was an increase of IL-1 $\beta$ level.

Nucleotide-binding domain leucine-rich repeats, pyrin domain-containing 3 (NLRP3) is a pattern recognition receptor, and will be activated by infection or various stress could form NLRP3 inflammasome after recruiting domain (CARD) domain (ASC) in combination with activated caspase-1. Previous experiments have shown that NLRP3 inflammasome activation occurs within HCECs in DED animal models induced by environment control of and clinical patients $[6,7,20]$. We further confirmed NLRP3 inflammasome occurred during this progress by detecting NLRP3, caspase-1, and IL-1ßboth also in vivo and in vitro within our experiment model.

Combing the research published, lysosomal disruption, Calcium signaling and TXNIP and ER stress have been reported make contribution to NLRP3 inflammasome activation [21]. However, the role of noncoding RNA in this process is still poorly understood. Although most of the time, most ncRNAs are expressed at a stable and lower level than coding RNA, IncRNAs play important roles in many biological processes, including ocular diseases, such as open-angle glaucoma[22], diabetic retinopathy [11]. There have still many studies have shown that IncRNAs participate in the process of serve DED, such as Sjögren's syndrome, a kind of autoimmunity disease [23]. However little concern focus on if IncRNA involved in DED triggered by other nonimmunologic factor.

KCNQ10T1 located on chromosome 11p15.5, can participate in and regulate the occurrence of various diseases, the expression level may has positive correlation with cancer stage [24], induce drug resistance[25], promoted tumor cell proliferation, migration and invasiveness [26]. Different from the human body benefiting from the induced cell death required by tumors, the premature programmed cell death in eye diseases may lead to the occurrence of diseases. For example, during the formation of cataracts, the expression of KCNQ10T1 is up-regulated, thus promotes the death of human crystal epithelial cells [27]. There is also evidence that in diabetic keratopathy, the corneal endothelium be in the high-glucose environment also has the high level of KCNQ10T1 expression, accompanied by the corneal endothelial cells decompensation, corneal dysfunction and edema occur, which further affects the vision [28].

There was still no direct evidence that KCNQ10T1 was involved in the course of DED, we proved through this experiment that it was correlated with the occurrence of DED, and the upregulation were observed in both cell and animal model of DED. KCNQ10T1 knock down can reduce the existence of pyroptosis, and promote the accelerated cell migration, which is an important factor of corneal epithelial recovery.

IncRNA has been believed can prevent target microRNA from binding with downstream mRNA translated into protein as posttranscriptional regulation. At the same time, by inquiring the database, we know that miR214 is the downstream gene of KCNQ10T1 as well, miR-214 plays a role in the modulation of 
vascular endothelial cell angiogenesis, proliferation, and senescence[29], also negatively regulates the sensitivity of tumor cells to chemotherapeutic drugs as a post-transcriptional regulatory factor [30].

After the intervention of KCNQ10T1, the level of miR-214 expression was down-regulated. While the increase of the miR-214 expression cannot reverse the content of KCNQ10T1. Meanwhile, the low expression of miR-214 could have antagonism effect with KCNQ10T1 knock down on the migration.

we also found the transcriptional level of miR-214 was significantly influence the NLRP3 inflammsome activation and IL-1 $\beta$ release, and combining the results come from bioinformatics calculation, caspase-1 come to be the key gene influenced by miR-214 varition when pyroptosis occur which has the highest matching degree.

While caspase-1, belongs to the aspartate cysteine transporter, as classical executor aggravate pyroptosis, could be activated by NLRP3 inflammasome[31, 32], and recruits the punching protein GSDMD[33], leading of inflammatory factors and other contents to release [34], therefore accelerating cell death [35]. The gain and loss experiments confirmed the relationship between miR-214 and caspase-1.

The interactive relationship between KCNQ10T1 and miR-214, miR-214 and casapse-1, KCNQ10T1 and caspase-1 were proved by our experiments.

In conclusion, it is speculated that KCNQ10T1 promotes the activation of inflammasomes in DED through inhibiting miR-214, indirectly increasing the activity of NLRP3 inflammasomes. And our experiments verified that siKCNQ10T1 can reduce NLRP3 inflammasome activation and promotes migration of human corneal epithelial cells and accelerate cell wound healing via lower the expression of miR-214 during dry eye disease.

Taken together, these results provide a hypothesis that we can design drugs that target RNA as new strategies for prevention and treatment in the future.

\section{Conclusions}

In conclusion, KCNQ10T1/miR-214/caspase1 axis played a vital role in the progression of DED, suggesting that KCNQ10T1 may be a potential target for the treatment of dry eye disease.

\section{Declarations}

*Author contribution

All authors contributed to the study conception and design. Material preparation, data collection and analysis were performed by [xuran Li], [yanyan Zhang], [xinyue Li] and [yu Yu]. The first draft of the manuscript was written by [xuran Li] and all authors commented on previous versions of the manuscript. All authors read and approved the final manuscript. 
*Consent to Participate (Ethics)

Not applicable.

*Consent to Publish (Ethics)

Not applicable. Availability of data and material

\section{Competing interests}

The authors declare that they have no competing interests"]

\section{Acknowledgements}

Not applicable.

\section{References}

1. Messmer, E.M., The pathophysiology, diagnosis, and treatment of dry eye disease. Dtsch Arztebl Int, 2015. 112(5): p. 71-81; quiz 82.

2. Roda, M., et al., Dry Eye Disease and Tear Cytokine Levels-A Meta-Analysis. Int J Mol Sci, 2020. 21(9).

3. Vande Walle, L. and M. Lamkanfi, Pyroptosis. Curr Biol, 2016. 26(13): p. R568-r572.

4. Shi, J., W. Gao, and F. Shao, Pyroptosis: Gasdermin-Mediated Programmed Necrotic Cell Death. Trends Biochem Sci, 2017. 42(4): p. 245-254.

5. Zhong, Z., E. Sanchez-Lopez, and M. Karin, Autophagy, NLRP3 inflammasome and autoinflammatory/immune diseases. Clin Exp Rheumatol, 2016. 34(4 Suppl 98): p. 12-6.

6. Zheng, Q., et al., Reactive oxygen species activated NLRP3 inflammasomes prime environment-induced murine dry eye. Exp Eye Res, 2014. 125: p. 1-8.

7. Zheng, Q., et al., Reactive oxygen species activated NLRP3 inflammasomes initiate inflammation in hyperosmolarity stressed human corneal epithelial cells and environment-induced dry eye patients. Exp Eye Res, 2015. 134: p. 133-40.

8. Peng, W.X., P. Koirala, and Y.Y. Mo, LncRNA-mediated regulation of cell signaling in cancer. Oncogene, 2017. 36(41): p. 5661-5667.

9. Liao, K., et al., The research progress of LncRNA involved in the regulation of inflammatory diseases. Mol Immunol, 2018. 101: p. 182-188.

10. Zhao, Z., et al., Mechanisms of IncRNA/microRNA interactions in angiogenesis. Life Sci, 2020. 254: p. 116900. 
11. Yan, B., et al., IncRNA-MIAT regulates microvascular dysfunction by functioning as a competing endogenous RNA. Circ Res, 2015. 116(7): p. 1143-56.

12. Chen, X., et al., LnCRNA ZNF503-AS1 promotes RPE differentiation by downregulating ZNF503 expression. Cell Death Dis, 2017. 8(9): p. e3046.

13. Bai, Y.H., et al., LnCRNA NEAT1 promotes inflammatory response and induces corneal neovascularization. J Mol Endocrinol, 2018. 61(4): p. 231-239.

14. Lu, J., F. Xu, and H. Lu, LncRNA PVT1 regulates ferroptosis through miR-214-mediated TFR1 and p53. Life Sci, 2020. 260: p. 118305.

15. Nosalski, R., et al., T-Cell-Derived miRNA-214 Mediates Perivascular Fibrosis in Hypertension. Circ Res, 2020. 126(8): p. 988-1003.

16. Bron, A.J., V.E. Evans, and J.A. Smith, Grading of corneal and conjunctival staining in the context of other dry eye tests. Cornea, 2003. 22(7): p. 640-50.

17. Yang, F., et al., Silencing long non-coding RNA Kcnq1ot1 alleviates pyroptosis and fibrosis in diabetic cardiomyopathy. Cell Death Dis, 2018. 9(10): p. 1000.

18. Aluru, S.V., et al., Tear Fluid Protein Changes in Dry Eye Syndrome Associated with Rheumatoid Arthritis: A Proteomic Approach. Ocul Surf, 2017. 15(1): p. 112-129.

19. Nair, M.G., et al., Goblet cell-derived resistin-like molecule beta augments CD4+ T cell production of IFN-gamma and infection-induced intestinal inflammation. J Immunol, 2008. 181(7): p. 4709-15.

20. Park, B., et al., Polydatin Inhibits NLRP3 Inflammasome in Dry Eye Disease by Attenuating Oxidative Stress and Inhibiting the NF-KB Pathway. Nutrients, 2019. 11(11).

21. Elliott, E.I. and F.S. Sutterwala, Initiation and perpetuation of NLRP3 inflammasome activation and assembly. Immunol Rev, 2015. 265(1): p. 35-52.

22. Lv, Y., et al., IncRNA TGF $\beta 2-A S 1$ promotes ECM production via TGF- $\beta 2$ in human trabecular meshwork cells. Biochem Biophys Res Commun, 2020. 527(4): p. 881-888.

23. Xin, M., et al., Mirt2 functions in synergy with miR-377 to participate in inflammatory pathophysiology of Sjögren's syndrome. Artif Cells Nanomed Biotechnol, 2019. 47(1): p. 2473-2480.

24. Liu, X., et al., Long non-coding RNA KCNQ10T1 up-regulates CTNND1 by sponging miR-329-3p to induce the proliferation, migration, invasion, and inhibit apoptosis of colorectal cancer cells. Cancer Cell Int, 2020. 20: p. 340.

25. Zheng, Z.H., et al., LnCRNA KCNQ1OT1 is a key factor in the reversal effect of curcumin on cisplatin resistance in the colorectal cancer cells. Mol Cell Biochem, 2020. 
26. Duan, Q., et al., IncRNA KCNQ10T1 knockdown inhibits colorectal cancer cell proliferation, migration and invasiveness via the PI3K/AKT pathway. Oncol Lett, 2020. 20(1): p. 601-610.

27. Jin, X., et al., Long Non-Coding RNA KCNQ10T1 Promotes Cataractogenesis via miR-214 and Activation of the Caspase-1 Pathway. Cell Physiol Biochem, 2017. 42(1): p. 295-305.

28. Zhang, Y., et al., Long noncoding RNA KCNQ10T1 induces pyroptosis in diabetic corneal endothelial keratopathy. Am J Physiol Cell Physiol, 2020. 318(2): p. C346-c359.

29. Chen, Y.L., et al., MicroRNA-214 modulates the senescence of vascular smooth muscle cells in carotid artery stenosis. Mol Med, 2020. 26(1): p. 46.

30. $\mathrm{Xu}, \mathrm{H}$., et al., Methylation-mediated miR-214 regulates proliferation and drug sensitivity of renal cell carcinoma cells through targeting LIVIN. J Cell Mol Med, 2020. 24(11): p. 6410-6425.

31. Guey, B., et al., Caspase-1 autoproteolysis is differentially required for NLRP1b and NLRP3 inflammasome function. Proc Natl Acad Sci U S A, 2014. 111(48): p. 17254-9.

32. Sollberger, G., et al., Caspase-1: the inflammasome and beyond. Innate Immun, 2014. 20(2): p. 115-25.

33. Gaidt, M.M. and V. Hornung, Pore formation by GSDMD is the effector mechanism of pyroptosis.

EMBO J, 2016. 35(20): p. 2167-2169.

34. Miao, E.A., J.V. Rajan, and A. Aderem, Caspase-1-induced pyroptotic cell death. Immunol Rev, 2011. 243(1): p. 206-14.

35. Shi, J., et al., Cleavage of GSDMD by inflammatory caspases determines pyroptotic cell death. Nature, 2015. 526(7575): p. 660-5.

\section{Figures}



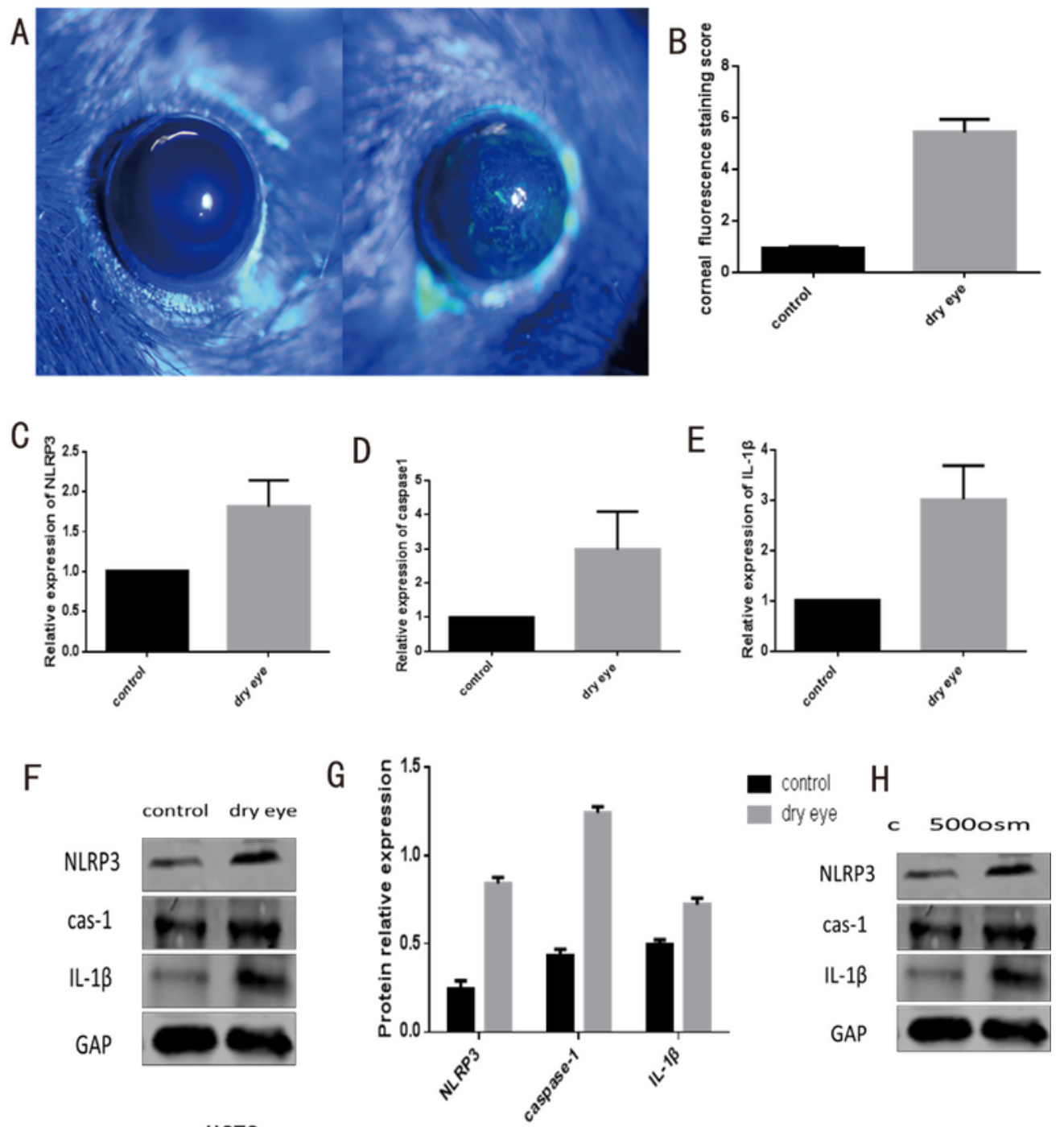

HCEC
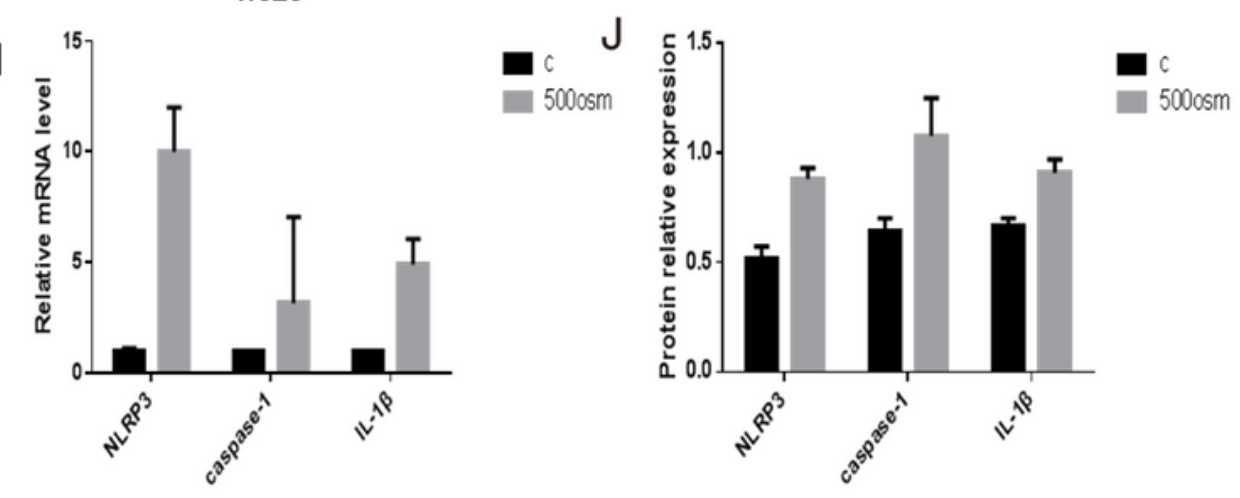

\section{Figure 1}

Expression of NLRP3 inflammasome and IL-1 $\beta$ in HCECs and cornea tissue A- B: The cornea tissue of each of five SD rats 160-180 g after subcutaneous injection with or without scopolamine hydrobromide were stained with $1 \%$ sodium fluorescein, and examined with a slit lamp microscope. A contrast between control rat cornea and dry eye cornea was shown C-E: cornea tissues mRNA were acquired after rats get injection with scopolamine hydrobromide for continuous fourteen days, amplified NLRP3, caspase-1 and 
IL-1ßmRNA products were normalized to GAPTH. Values are expressed as the means \pm SD from three independent biological replicates. F-G: Whole protein of corneas electrophoresis were taken on $12.5 \%$ polyacrylamide gels with sodium dodecyl sulfate-polyacrylamide gel electrophoresis (SDS-PAGE) and immunoblotted with an anti- NLRP3 antibody, anti- caspase-1 antibody, anti- IL-1 $\beta$ antibody and anti- $\beta$ actin antibody. Ratios of $\beta$-actin are shown as relative intensities. Values are expressed as means \pm SD from three independent biological replicates $\mathrm{H}-\mathrm{J}$ : After treated with cultured medium with $500 \mathrm{osm}$ NACL for $24 \mathrm{~h}$, whole-cell lysates were fractionated on $12.5 \%$ polyacrylamide gels with SDS-PAGE and immunoblotted with anti- NLRP3 antibody, anti- caspase-1 antibody, anti- IL-1 $\beta$ antibody and anti- $\beta$-actin antibody. Ratios of HIF-1 $\alpha / \beta$-actin are shown as relative intensities. Values are expressed as means \pm SD from three independent biological replicates. I: HCECs were cultured for 4 hours with 500 osm NACL contained medium. Gene expression change was determined by PCR, The related gene expression levels were high, while that of those with lower expression levels in control HCECs 

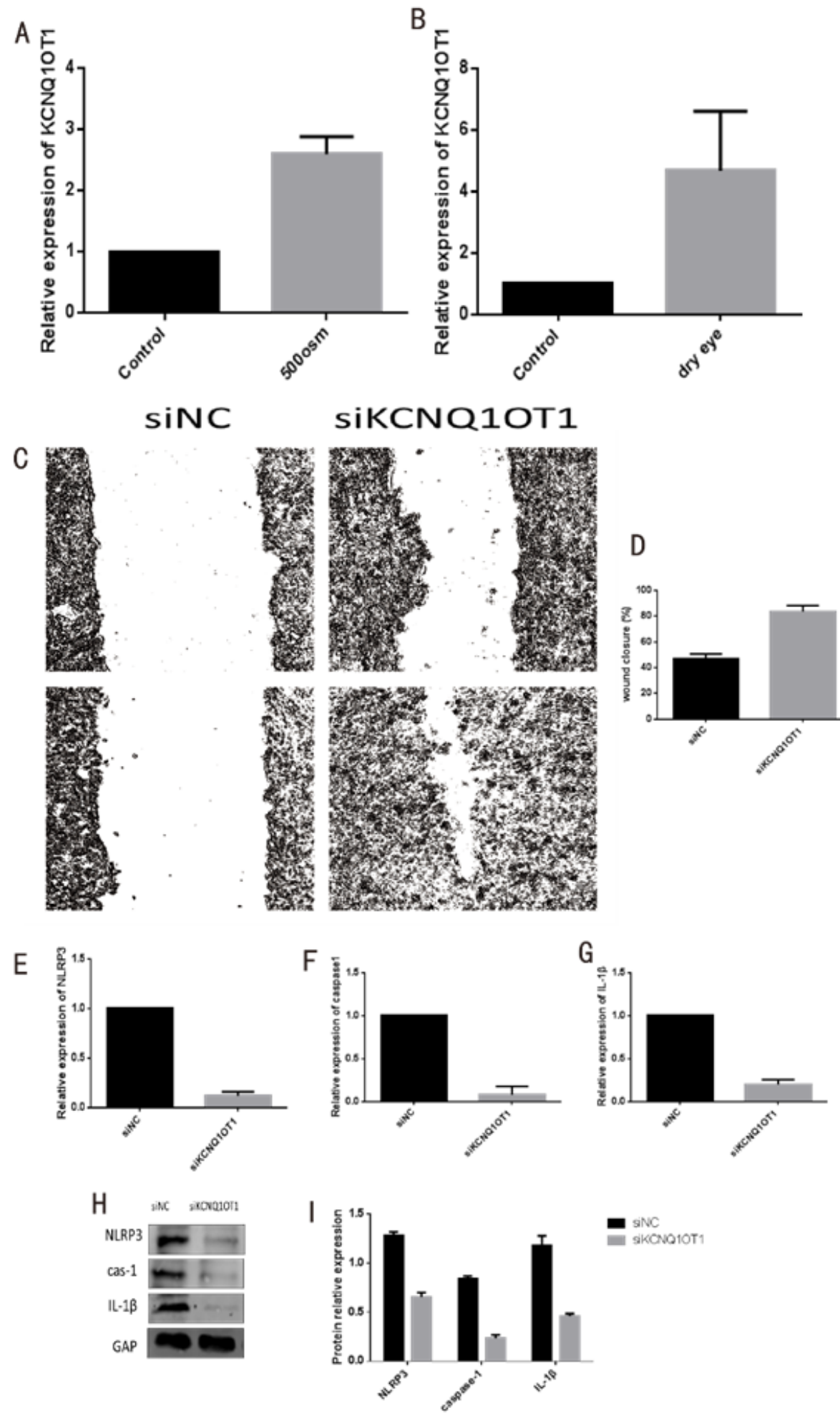

Figure 2

KCNQ10T1-knock down HCECs failed to activate NLRP3 inflammmasome A: cornea tissues total RNA were acquired after rats induced dry eye successfully, the expression of KCNQ10T1 was up regulated after normalized by GAPDH. Values are expressed as the means \pm SD from three independent $b$ iological replicates. B: after HCECs cultured within hyperosmosis medium or not. Gene expression change of KCNQ10T1 was determined by the means \pm SD from three independent biological replicates C-D: after 
HCECs were cultured within hyperosmosis medium or not, extracellular flotage were removed from culture medium. Cell migrate ability was monitored by cell starch assay with the microscope system after KCNQ10T1 knock down. The remaining area rate was used to evaluate the influence of KCNQ1OT1. Values are expressed as the means \pm SD from four independent biological replicates. The statistical significance of differences was calculated by ANOVA followed by Student's t-test. E-G: after HCECs were cultured within hyperosmosis medium or not, extracellular flotage were removed from culture medium. Whole mRNA of HCECs were determined by PCR to evaluate the influence come from KCNQ10T1 knock down, and NLRP3, caspase-1 and IL-1ßproducts were normalized to GAPDH. Values are expressed as the means \pm SD from three independent biological replicates. $\mathrm{H}$-l: whole-cell lysates extracted from KCNQ10T1 knock down HCECs were fractionated on 12.5\% polyacrylamide gels with SDS-PAGE and immunoblotted with anti- NLRP3 antibody, anti- caspase- 1 antibody, anti- IL- $1 \beta$ antibody and anti- $\beta$-actin antibody. Ratios of $\beta$-actin are shown as relative intensities. Values are expressed as means \pm SD from three independent biological replicates. 

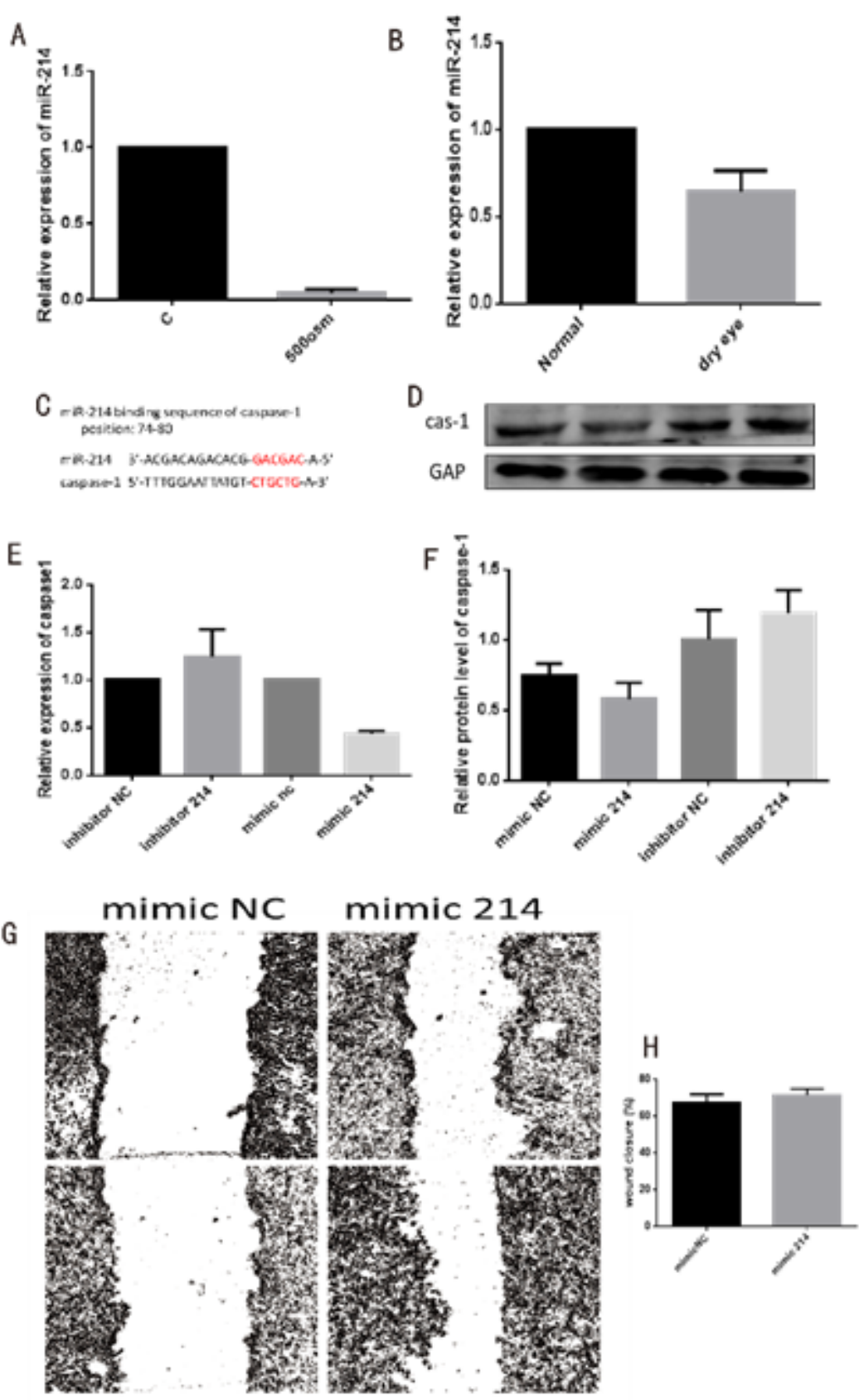

Figure 3

miR-214 overexpression makes HCECs failed to activate NLRP3 inflammmasome A: cornea tissues total RNA were acquired after rats induced dry eye successfully, the expression of miR-214was up regulated after normalized by GAPTH. Values are expressed as the means \pm SD from three independent $b$ iological replicates. B: after HCECs cultured within hyperosmosis medium or not. Gene expression change of miR214 was determined by the means \pm SD from three independent biological replicates $\mathrm{C}$ : The bioinformatic prediction for the binding sites between caspase-1 and miR-214. E: the potent correlation between miR-214 and caspase-1 was determined by PCR, after HCECs were treated by miR-214 mimics and miR-214 inhibitors and their corresponding negative controls, Whole mRNA of HCECs were determined by PCR to evaluate the influence come from KCNQ10T1 knock down, and NLRP3, caspase-1 and IL-1ßproducts were normalized to GAPDH. Values are expressed as the means \pm SD from three 
independent biological replicates. D-F: the potent correlation between miR-214 and caspase-1 was determined by SDS-PAGE, after HCECs were treated by miR-214 mimics and miR-214 inhibitors and their corresponding negative controls, whole-cell lysates were extracted and fractionated on $12.5 \%$ polyacrylamide gels with SDS-PAGE and immunoblotted with anti- NLRP3 antibody, anti- caspase-1 antibody, anti- IL-1 $\beta$ antibody and anti- $\beta$-actin antibody. Ratios of $\beta$-actin are shown as relative intensities. Values are expressed as means \pm SD from three independent biological replicates. G-I: after HCECs were cultured within hyperosmosis medium or not, extracellular flotage were removed from culture medium. Cell migrate ability was monitored by cell starch assay with the microscope system after miR-214 overexpression. The remaining area rate was used to evaluate the influence of KCNQ10T1. Values are expressed as the means \pm SD from four independent biological replicates. The statistical significance of differences was calculated by ANOVA followed by Student's t-test.

A

miR-214 binding sequence of KCNQ1OT1 position: 31761-31769

miR-214 3'-TGACGGACAGAC-ACGGACGAC-A-5' KCNQ1OT1 5'-TGGGAGGATGCC-TGCCTGCTG-G-3'
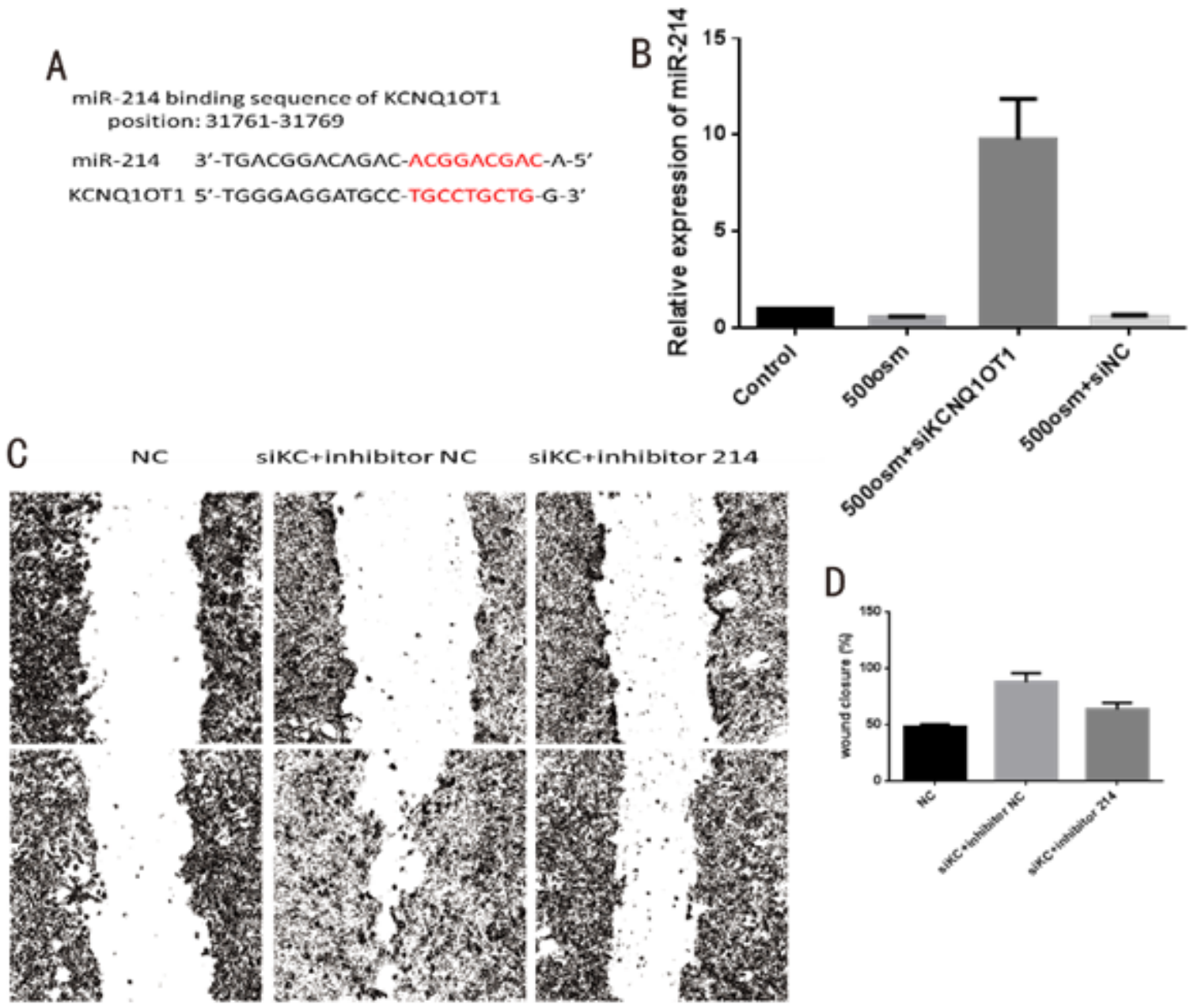

Figure 4

the relationship between KCNQ10T1 and miR-214 A: The bio-informatic prediction for the binding sites between KCNQ10T1 and miR-214. B: After HCECs or those KCNQ10T1 knock down were cultured within hyperosmosis medium or not for $4 \mathrm{~h}$, total RNA were extracted and determined by PCR to evaluate the influence on miR-214 come from KCNQ10T1 knock down, products were normalized to U6. Values are expressed as the means \pm SD from three independent biological replicates. C-D: HCECs were transfected 
with siNC +miR-NC, siKCNQ10T1+miR-NC, siKCNQ10T1+miR-214 inhibitors then cell wound ratio was detected by cell migrate rate. Data represent the means \pm SD of four independent biological replicates. The statistical significance of differences 